\title{
Esa diaria gotera de la muerte: Mortalidad infantil y los albores de la medicina pediátrica en la provincia de Santiago, Chile, 1870-1913
}

\author{
That daily trickle of death: Infant mortality and the dawn \\ of pediatric medicine in the province of Santiago, Chile, \\ 1870-1913
}

${ }^{1}$ Autor de correspondencia. Doctor en Historia. Programa de Doctorado en Historia. Universidad de Chile: Santiago, Chile. $\otimes$ iD

${ }^{2}$ Doctor en Historia. Profesor Adjunto e Investigador, Centro de Estudios Históricos, Universidad Bernardo O'Higgins; Santiago, Chile. $\bowtie$ (iD)
RESUMEN Este artículo aborda el problema de la mortalidad infantil en la provincia de Santiago, Chile, y el desarrollo de la medicina pediátrica durante la segunda mitad del siglo XIX e inicios del XX, a través de especialistas que contribuyeron a organizar los conocimientos y prácticas que estructuraron su ejercicio profesional. Para emprender este objetivo y sistematizar este nuevo campo médico, se analizan los antecedentes relativos a la formación de la Facultad de Medicina y el quiebre que representó la aparición de una asignatura dedicada a las enfermedades de la niñez. La investigación se encuentra respaldada en diversas fuentes históricas, entre ellas, la prensa escrita, bibliografía médica, memorias de titulación y el Anuario Estadístico de la República de Chile. La inauguración de los hospitales de niños expresa una institucionalidad, en la que los facultativos posicionaron su labor, pusieron en práctica los tratamientos científicos y, lo más importante, se convirtieron en espacios que permitieron la disminución de la mortalidad infantil.

PALABRAS CLAVES Mortalidad Infantil; Pediatría; Servicios de Salud del Niño; Chile.

\begin{abstract}
This article deals with the problem of infant mortality in Santiago, Chile, and the development of pediatric medicine during the second half of the nineteenth century and the beginning of the twentieth century. Emphasis is placed on the specialists who contributed to organizing the knowledge and practices that structured their professional field. In order to pursue the objective and systematize this new medical field, our analysis suggests the decisive role of the establishment of the Faculty of Medicine and the appearance of coursework dedicated specifically to childhood diseases. Our research is based on various historical sources including the press, medical literature, thesis archives, and the Anuario Estadístico de la República de Chile [Statistical Yearbook of the Republic of Chile]. Likewise, the inauguration of children's hospitals expresses an institutional setting where physicians position their work, put scientific treatments into practice, and most importantly, they become spaces that allow for the reduction of infant mortality.
\end{abstract}

KEY WORDS Infant Mortality; Pediatrics; Child Health Services; Chile. 


\section{INTRODUCCIÓN}

Según las cifras publicadas el año 2014 por el Instituto Nacional de Estadística (INE) ${ }^{(1)}$, la tasa bruta de mortalidad neonatal en la provincia de Santiago era de 4,8 por 1.000 nacidos vivos y las defunciones de menores de un año eran de 6,8 por 1.000 nacidos vivos. Las cifras nacionales muestran tendencias similares: a nivel país, la tasa de mortalidad neonatal fue de 5,2 por 1.000 nacidos vivos y las defunciones de menores de un año fue de 7,2. Los valores exhibidos coinciden con la información emanada de la Comisión Económica para América Latina y el Caribe (CEPAL), que deja al país con uno de los índices más bajos de fallecimientos en América Latina. En el Anuario Estadístico de América Latina y el Caribe elaborado por la CEPAL, de los 35 países considerados en la medición, Chile aparece en el segundo lugar, superado por Cuba con una mortalidad infantil de 5,0 por cada 1.000 nacidos vivos ${ }^{(2)}$. Estos números difieren, absolutamente, de los registrados durante la segunda mitad del siglo XIX y las primeras décadas del siglo XX, cuando Chile era considerado la nación con la mayor mortalidad infantil a escala mundial. Estas dos realidades opuestas se convierten en un desafío para entender el cambio histórico que ocasionó el proceso de medicalización de larga duración y estudiar las variables que explican una transformación demográfica de carácter revolucionario.

Las investigaciones sobre la mortalidad infantil y sus relaciones con la pediatría han sido tratadas por médicos e historiadores. Los facultativos profundizan la función de los especialistas, que aparecen como parte de una ciencia con progresos constantes y reforzando los antecedentes biográficos ${ }^{(3,4,5,6)}$. A medida que avanza el siglo XX, los contenidos adquieren un elevado grado de especialización y complejidad ${ }^{(7,8)}$. Por su parte, los historiadores se han aproximado a los estudios de la infancia construyendo históricamente la niñez y las condiciones de vida paupérrimas experimentadas por los niños. En la perspectiva cultural, se tratan las modificaciones de los sentimientos maternos hacia los hijos que se expresan en cuidados y tratamientos frente a una enfermedad ${ }^{(9,10)}$. Estas propuestas sostienen que los procesos económicos y médicos fracasaron en la intención de disminuir la elevada mortalidad infantil. Situación que, más avanzado el siglo veinte, evidencia una transformación y mejoramiento que reduce las cifras mortales ${ }^{(11,12,13,14,15)}$. Sin embargo, todavía quedan espacios e interrogantes para profundizar en la problemática de la mortalidad infantil y sus asociaciones con el desarroIlo de la medicina pediátrica en Chile.

La historiografía chilena ha trabajado la configuración de las profesiones médicas, la difusión de saberes en espacios más amplios que la academia y la medicalización en la infancia( ${ }^{(16,17,18)}$. Lo ha hecho a partir de dos circuitos simultáneos: la divulgación y apropiación de los saberes europeos, por un lado, y el conocimiento local de la población analizada, por el otro. El estudio de los procesos de especialización dentro de las profesiones, se vincula con los cambios tecnológicos y la organización política que apoyó las actividades de los doctores ${ }^{(19)}$. Por esta razón, resulta fundamental indagar en la función de los médicos durante el surgimiento de una nueva especialidad, la validez de los diagnósticos y la aplicación de los tratamientos propuestos en los hospitales.

\section{ASPECTOS METODOLÓGICOS}

La línea de trabajo de esta investigación aborda la formación de la profesionalización de la medicina pediátrica, mediante conocimientos científicos disciplinarios. Para sustentar esta afirmación existen dos trayectorias de estudio. Por un lado, la mirada tradicional del fenómeno tiende a reforzar el quehacer de los médicos desde un punto de vista unidireccional, o sea, el sabio que juega un rol activo, y otro actor que solo acata los preceptos. Este énfasis aprecia los avances como una sumatoria de saberes, el diálogo entre especialistas y la adquisición de experticia en el laboratorio. Por otro lado, la renovación de este enfoque 
por la influencia de la historia cultural, ha ampliado y enriquecido las interpretaciones del pasado médico ${ }^{(20)}$, esta perspectiva explora las rutas y los significados de la profesionalidad, relacionadas con el sistema sociocultural en que se encuentra anclado ${ }^{(21)}$. Así, discute los procedimientos que han ocupado las disciplinas para validarse en un contexto determinado y los métodos empleados para legitimar los tratamientos médicos ${ }^{(22,23,24,25)}$.

Metodológicamente, el estudio de las fuentes documentales permite desarrollar una crítica que se aplica a una serie de interrogantes con el propósito de evaluar los significados, explicaciones y prácticas en torno a la mortalidad infantil ${ }^{(26,27)}$. Este método es clave para interpretar el contenido de las fuentes y, de esa manera, comprender el contexto de producción asignado, la perspectiva de los autores y los tránsitos de los saberes. En ese marco, las publicaciones de prensa utilizadas provienen de varios títulos, que informan sobre la mortandad, el impacto de las afecciones a nivel social y los tratamientos médicos. También, los trabajos científicos de la materia abordan la configuración de los conocimientos, las discusiones en las cátedras médicas y la implementación de nuevas salas en el sistema hospitalario. Este cuerpo documental es complementado con el Anuario Estadístico, registro que detecta la evolución demográfica y médica en la población.

En este texto se abordan dos hitos para la configuración de los conocimientos pediátricos: la fundación de estudios universitarios dedicados a las afecciones en la niñez y la instalación de espacios clínicos dedicados a la práctica de estos saberes. Se considera como punto inicial de la problemática, la creación de la cátedra de enfermedades de la infancia en 1870, luego continúa con la instalación del hospital provisorio de niños en 1901 (futuro Roberto del Río) y concluye con la inauguración del Hospital Manuel Arriarán en 1913, especializado en las afecciones de la niñez. La ejecución de ambas acciones corresponde a dos hechos históricos fundamentales para la articulación de los engranajes médicos que permitieron reducir la mortalidad infantil. A partir de estos cimientos, los médicos consolidaron las recomendaciones y los tratamientos. La existencia de estos recintos permite difundir contenidos, a través de la prensa y literatura, cambios en los cuidados de los recién nacidos, mejorar las prácticas de lactancia y construir la medicación de los niños.

Este artículo es parte de los resultados de la tesis doctoral "Esa diaria gotera de la muerte: mortalidad infantil en Santiago (1860-1914)" realizada en el marco del programa del Doctorado en Historia de la Universidad de Chile.

\section{LA MORTALIDAD INFANTIL EN CIFRAS}

Para calcular la tasa de mortalidad infantil se consideraron las anotaciones de nacimientos y defunciones realizadas por los párrocos (1848-1884) y por el Registro Civil (18851930). El proceso de clasificación de ambas instituciones fue distinto: la primera consideró los fallecimientos hasta los siete años; en cambio, la segunda estimó las muertes entre el nacimiento y el año de vida. La fórmula utilizada abordó la relación entre las defunciones de los niños (hasta 1 o 7 años) y el total de nacidos vivos durante un año. Cabe señalar que las categorías de los eclesiásticos generan un obstáculo en los resultados, dado que la mortalidad infantil tenía efectos diferentes de acuerdo con la etapa del infante, por lo que el índice disminuía a medida que la edad del niño aumentaba. En el período neonatal (primeras cuatro semanas), las probabilidades de fallecer eran altísimas. Siguiendo un criterio etiológico, entre las causales, predominaron las de carácter endógenas, ellas eran: inmadurez del recién nacido, malformación o debilidad congénita. A continuación, la fase post neonatal (entre uno y doce meses), las posibilidades de morir eran altas, pero menores respecto a la época anterior, manifestadas fundamentalmente por causas de tipo exógenas, entre ellas: enfermedades infecciosas, desnutrición y alimentación inadecuada. Esta división, planteada por Victoria Mazzeo, permite reflexionar sobre las estimaciones de la 
mortalidad infantil según la edad y las causas de muerte. Sin embargo, establecer este modelo no representó una actuación determinada en la práctica. Un infante que nació con debilidad congénita (endógena), tuvo mayores probabilidades de contraer una enfermedad de tipo infecciosa (exógena) ${ }^{(28)}$. Respecto de la alimentación inadecuada, el doctor Calvo Mackenna puso en evidencia la magnitud de este fenómeno: "si juntamos diez niños que hayan muerto antes de cumplir un año, a consecuencia de enfermedades del intestino, encontraremos con seguridad que por lo menos ocho de ellos tomaban mamaderas de leche de vaca o cualquier otro alimento"(29).
La Tabla 1 presenta una tasa de mortalidad infantil con tendencias de valores altos. Tras comparar estos registros demográficos con las principales capitales del continente, los médicos concluyeron que "Chile es uno de los países de América que ofrece la mayor mortandad infantil"(30); entre los años 1848 y 1885, más de 400 de cada 1.000 niños fallecían antes de superar la etapa consignada como infancia (hasta los siete años). Si bien con la instalación del Registro Civil la categoría sufrió modificaciones y comenzó a registrar la mortalidad infantil hasta un año, las cifras continuaron marcando valores altísimos, por encima de las 300 muertes de cada

Tabla 1. Número de nacimientos y fallecimientos y tasa de mortalidad infantil (por 1.000 nacidos vivos), provincia de Santiago, Chile, 1848-1930.

\begin{tabular}{|c|c|c|c|c|c|c|c|}
\hline Año & Nacimientos & Fallecimientos & $\begin{array}{l}\text { Tasa de } \\
\text { mortalidad } \\
\text { Infantil }\end{array}$ & Años & Nacimientos & Fallecimientos & $\begin{array}{c}\text { Tasa de } \\
\text { mortalidad } \\
\text { Infantil }\end{array}$ \\
\hline 1848 & 9.551 & 3.856 & 403,7 & 1881 & 18.432 & 7.924 & 430,0 \\
\hline 1849 & 10.361 & 3.869 & 373,4 & 1882 & 18.256 & 8.207 & 449,6 \\
\hline 1850 & 9.880 & 4.022 & 408,0 & 1883 & 18.014 & 7.313 & 406,0 \\
\hline 1851 & 11.851 & 3.642 & 307,3 & 1884 & 14.235 & 4.308 & 302,6 \\
\hline 1852 & 11.330 & 5.568 & 491,4 & 1885 & 5.390 & 2.488 & 461,6 \\
\hline 1853 & 12.887 & 6.304 & 489,2 & 1886 & 4.674 & 1.482 & 317,1 \\
\hline 1854 & 12.813 & 5.183 & 404,5 & 1887 & 4.744 & 1.544 & 325,5 \\
\hline 1857 & 13.470 & 6.182 & 459,0 & 1888 & 7.526 & 2.868 & 381,1 \\
\hline 1858 & 14.783 & 5.792 & 391,8 & 1889 & 14.912 & 5.181 & 347,4 \\
\hline 1860 & 15.512 & 7.711 & 497,1 & 1896 & 8.523 & 4.093 & 480,2 \\
\hline 1861 & 14.806 & 6.565 & 443,4 & 1909 & 18.602 & 8.316 & 447,0 \\
\hline 1862 & 14.268 & 6.969 & 488,4 & 1912 & 21.209 & 8.339 & 393,2 \\
\hline 1863 & 15.136 & 8.406 & 555,4 & 1913 & 21.720 & 8.029 & 369,7 \\
\hline 1864 & 15.219 & 9.069 & 595,9 & 1915 & 21.803 & 7.193 & 330,0 \\
\hline 1865 & 13.636 & 7.323 & 537,0 & 1916 & 23.632 & 6.846 & 289,7 \\
\hline 1866 & 15.313 & 6.731 & 439,6 & 1917 & 25.278 & 7.863 & 311,1 \\
\hline 1867 & 15.629 & 6.959 & 445,3 & 1918 & 24.689 & 7.510 & 304,2 \\
\hline 1868 & 15.530 & 6.282 & 404,5 & 1919 & 24.081 & 8.990 & 373,3 \\
\hline 1869 & 16.015 & 9.332 & 582,7 & 1920 & 24.628 & 7.542 & 306,2 \\
\hline 1870 & 16.418 & 6.796 & 413,9 & 1921 & 26.489 & 9.376 & 354,0 \\
\hline 1872 & 16.319 & 7.718 & 473,0 & 1923 & 27.340 & 9.109 & 333,2 \\
\hline 1873 & 17.626 & 7.643 & 433,6 & 1924 & 28.904 & 8.559 & 296,1 \\
\hline 1874 & 16.748 & 7.368 & 439,9 & 1925 & 28.136 & 8.624 & 306,5 \\
\hline 1876 & 16.665 & 9.476 & 568,6 & 1926 & 29.760 & 8.511 & 286,0 \\
\hline 1877 & 16.109 & 7.744 & 480,7 & 1927 & 33.674 & 7.947 & 236,0 \\
\hline 1878 & 15.414 & 8.289 & 537,8 & 1928 & 39.896 & 6.936 & 173,9 \\
\hline 1879 & 17.340 & 8.487 & 489,4 & 1929 & 34.497 & 8.040 & 233,1 \\
\hline 1880 & 17.557 & 10.170 & 579,3 & 1930 & 34.842 & 8.667 & 248,8 \\
\hline
\end{tabular}

Fuente: Elaboración propia con base en los datos del Anuario Estadístico de la República de Chile, 1848-1930. 
1.000 nacidos vivos. Estos números, a partir de la década de 1920, comenzaron a descender por los efectos de múltiples factores, entre ellos: la introducción de la medicina infantil, los avances en salubridad, las mejoras y regulaciones sobre la alimentación. Esta trayectoria a la disminución no tuvo carácter abrupto, sino que manifestó períodos de reducción alternando con otros de crecimientos de las muertes, relacionados con algún brote infeccioso o las crisis socioeconómicas en esos años.

\section{LA CÁTEDRA DE MEDICINA INFANTIL}

Uno de los primeros textos que incluye el concepto pediatría es el tratado Poedoiatreia practica, T. Curatio morborum puerilium (1722) escrito por Theodor Zwinger III (1658-1724). Sin embargo, esta especialidad formó parte de la Obstetricia y la Medicina Interna hasta mediados del siglo siguiente. Por aquel entonces, se entendía que los organismos de los niños respondían igual que los adultos frente a las enfermedades, pero los estudios llevados a cabo en Francia y Alemania permitieron la separación paulatina y la profundización de un saber cuyo propósito era el cuidado, la crianza y la prevención de las enfermedades en la infancia. En Francia, el doctor Charles Michel Billard publicó, en 1828, el Traité des maladies des enfants nouveau-nés et a la mamele, estudio realizado a partir del registro de los niños internados en Le Hospices des Enfants Trouvés ${ }^{(31,32)}$. En las universidades alemanas de Berlín y Wurzburgo se fundaron, en 1858, las cátedras de Pediatría.

Los trabajos historiográficos sobre la medicina en Chile han abordado las dificultades que tuvo la configuración de los campos médicos, específicamente la ausencia de especialistas. Al respecto, el conocimiento médico científico impulsado bajo el alero de la Universidad de Chile y el Estado tuvo como precursores a expertos procedentes de Europa que aportaron sus habilidades para formar las primeras generaciones de pediatras. En 1833, el gobierno establece la enseñanza de la medicina en Chile, y en el Decreto de creación del curso señalaba que:

...deseando el Gobierno promover el
estudio de las ciencias médicas, que
aunque reconocido en todas las nacio-
nes del mundo como primera necesidad
para la conservación de la vida, ha sido
descuidado en Chile a influjo de una
preocupación vulgar [...] se abrirá en el
Instituto Nacional un curso de ciencias
médicas que durará seis años.

La organización de esta enseñanza comprendía cuatro secciones: anatomía, farmacia, medicina y cirugía. Sin embargo, este plan de estudios de 1833 experimentó varias complicaciones en su implementación, entre ellas: el reducido número de profesores, la falta de clases en los hospitales hasta la década de 1840 y la extensión de las etapas de formación de los estudiantes que duraban en promedio diez años. A raíz de estos problemas, las autoridades universitarias llevaron a cabo sucesivas reformas en los años 1845, 1860 y 1868, principalmente, a la estructura de las materias y la inclusión o modificación de algunos contenidos.

Durante la década de 1860, los médicos criticaban la escasa preocupación sobre la mortalidad infantil. A juicio de los especialistas, los médicos no sabían curar las enfermedades de los niños. Cada mujer tenía la potestad para estimar el mejor tratamiento para los infantes enfermos y en caso de no encontrar respuestas "recurre a tal o cual charlatán de conocida reputación; por manera que con tan intelijentes personas no es duro creer que las enfermedades triunfen casi siempre" ${ }^{\prime \prime(34)}$. Cuando llegaba el turno de consultar a uno de los escasos médicos especializados en medicina general, era demasiado tarde. Así, la base institucional frágil, los diagnósticos erróneos y la formación universitaria de los médicos generaban conocimientos precarios en torno a las enfermedades de la niñez.

En 1868, la Facultad de Medicina inauguró la cátedra dedicada a las enfermedades mentales y nerviosas, con una sección práctica que consistía en la asistencia a la Casa de Locos. La 
enseñanza de estas materias tuvo una vigencia solo de dos años, pues en 1870 fue reemplazada por la cátedra sobre enfermedades de los niños. El primer profesor designado para impartir el curso fue el doctor Francisco Javier Tocornal, en ese momento encargado de las visitas médicas a la Casa de Huérfanos, quien desempeñó esta labor hasta su jubilación, en 1882. En sus inicios, las clases de esta asignatura reforzaron un enfoque teórico más que el trabajo práctico en clínica o laboratorios, a diferencia de lo que ocurría con otras materias como Medicina Legal, Anatomía o Farmacia que contaban con establecimientos como los hospitales de San Borja o San Juan de Dios, la morgue o la Casa de Locos, donde ejercían la práctica de estas áreas de estudio.

La reforma del plan de estudios médicos, en 1881, instalaba en el sexto año la asignatura de "clínica obstétrica, jinecolójica i de niños" ${ }^{\prime \prime(35)}$, que al estar ubicada al finalizar la carrera, sería cursada por alumnos con las herramientas adquiridas en los años anteriores. Cuatro años después, la Facultad de Medicina discutía modificaciones a esta estructura, tendientes a separar los ramos entre Obstetricia y clínica obstétrica (seis horas semanales) y la Clínica de enfermedades de niños (tres horas semanales), ambas dictadas en el sexto año de la carrera. El encargado de dictar este curso, entre 1888 y 1910, fue el doctor Roberto del Río, titulado como médico cirujano en la Universidad de Chile en 1883, quien se especializó en las afecciones infantiles. Su permanencia a cargo de esta materia, por más de dos décadas, le permitió dar continuidad al programa de estudios, configurar las bases de los conocimientos pediátricos y, su mayor aporte, consolidar la formación de los primeros especialistas de esta rama de la medicina en el país. La contribución del doctor del Río no solo se reduce a las aulas, sino que también jugó un papel fundamental en la fundación del Hospital de Niños, ya que estuvo a cargo de la administración del recinto, desde 1907 hasta su muerte una década más tarde.

El desarrollo de la cátedra de pediatría estuvo relacionado con la fundación de los hospitales de niños, que implicaba el ejercicio diario de la medicina, la aplicación de los tratamientos descritos en libros y la adquisición de experiencia técnica. Hasta ese momento, la falta de trabajo práctico de los especialistas en la clínica de niños era otra dificultad para sostener diagnósticos certeros de las enfermedades. En 1882, en su "Memoria de prueba para optar al grado de Licenciado en la Facultad de Medicina i Farmacia" Vargas describía que es "desesperante para un médico en los primeros años de su profesión la presencia de un párvulo enfermo; la mayor parte de las veces se vé obligado a no tomar medida alguna por carecer enteramente de práctica en las enfermedades que son comunes en ellos"(36). De igual manera, Stafford Mc Lean indicaba, en 1918, que "mientras menos libros de enseñanza pediátrica y más cursos prácticos, tanto mejor será la acción social de la puericultura" ${ }^{\prime(37)}$.

En 1914, Ismael Valdés, presidente del Consejo Superior de Protección a la Infancia, escribió al Decano de la Escuela de Medicina indicando que los alumnos obtenían el título profesional sin considerar, entre las pruebas de promoción o de especialidades, las del ramo de enfermedades de niños. Solo bastaba la asistencia reglamentaria por el año que duraban las clases lectivas, siendo luego sometidos a los exámenes finales de titulación. Dado que el curso se desarrollaba durante el sexto año, los estudiantes estaban más preocupados por la planificación de las pruebas que los habilitarían para ejercer su profesión, que por los cursos de pediatría. La autoridad destacaba la atención que requerían los estudios de esta disciplina con el propósito de evitar que "los jóvenes médicos salgan de la Escuela sin la preparación suficiente en una especialidad cuya enorme importancia justifica con exceso el alto porcentaje de nuestra mortalidad infantil"(38).

\section{LOS HOSPITALES}

Por el crecimiento demográfico en la provincia de Santiago durante la segunda mitad 
del siglo XIX, la Junta de Beneficencia agregó nuevos hospitales a los existentes de San Juan de Dios (1543) y San Borja (1776). Indudablemente, los gastos en profilaxis aumentaron proporcionalmente al incremento de los habitantes, razón por la cual los trabajos demandaban la colaboración económica de los vecinos, junto con los aportes de la beneficencia y las subvenciones estatales. En estas circunstancias, se construyeron los hospitales Vicente de Paul (1872), El Salvador (1872) y San José (1887). Aunque los proyectos de edificación fueron concluidos, la población siguió aumentando y, con ello, el número de enfermos anuales a fines de la centuria.

Hasta las primeras décadas del siglo XX, los hospitales atendían solo hombres y mujeres adultas. Desde luego, la falta de un hospital de niños les generaba dificultades a los médicos para conocer las morbilidades infantiles y no tenían dónde aplicar las perspectivas teóricas, acentuando la separación entre un campo que trataba de legitimarse y las personas. Por ello, es importante destacar que la falta de hospitales para las afecciones de los niños contribuyó al incremento de la mortalidad infantil. Si se tiene en cuenta la frecuencia con que se presentaban las enfermedades en los recién nacidos, la falta de un recinto hospitalario obligaba a los padres a procurar el restablecimiento de la salud de los infantes sin la colaboración de facultativos, por esa razón las autoridades políticas y los médicos indicaban la urgencia de fundar, en Santiago o Valparaíso, un hospital dedicado exclusivamente para la curación de niños menores de siete años.

Los dispensarios eran establecimientos donde los enfermos recibían asistencia médica y farmacéutica, una red de locales que apoyaban a la comunidad repartiendo alimento y vestuario entre los recién nacidos. Sin embargo, en la segunda mitad del siglo XIX, el crecimiento de la población ocasionó un deterioro de los servicios que ofrecían. Sumado a ello, la falta de medicamentos y la gran cantidad de personas que acudían generaron menos atención de los facultativos en los niños, y las aglomeraciones aumentaban las probabilidades de que los menores contrajeran infecciones.
Las madres eran quienes, principalmente, tomaban determinaciones sobre la salud de los recién nacidos, empleando diferentes métodos validados por la experiencia: reconocían, examinaban, definían y trataban los malestares, sustentando los tratamientos de la enfermedad en los consejos de las redes familiares y comunitarias. En esa línea, el doctor Jenaro Lisboa, observó la práctica popular que "Ilaman quebrar el empacho, acto de salvajismo sin mas esplicacion que la crasa ignorancia de los que los ejecutan"(39).

La mayoría de los párvulos que contraían una enfermedad terminaba, de manera adversa o favorable, sin recibir observaciones médicas y posiblemente sus padres tampoco se preocuparan por esta condición. Según el doctor Ricardo Dávila Boza:

[por] ignorancia, por desidia o por imposibilidad material, los padres de estos pobres niños no les prestan ninguna atención; los crían dejándolos abandonados a sus propias fuerzas, i cuando se enferman, lo más que suelen hacer es reprenderlos o amonestarlos; pero nunca se les ocurre siquiera echarlos a la cama. ${ }^{(40)}$

De allí se desprende que, entre los niños, cualquier brote infeccioso tenía como consecuencia graves estragos y dolencias que, si bien al principio eran leves, le ocasionaban la muerte y podrían haber sido curadas con la aplicación de un tratamiento en el momento oportuno.

Esto favoreció el uso de métodos medicinales no convencionales y muestra los múltiples límites de los conocimientos médicos científicos que, en este período, todavía eran permeables a planteamientos validados solo por la experiencia de las madres. Por su parte, los manuales recalcaban los cuidados que se debían tener en la administración de los medicamentos, sobre todo con las fórmulas químicas que podían causar daño: "el estómago de la criatura no lo soporta, o que se administra por ignorancia a dosis inconvenientes, o que se prepara mal"(41).

¿A partir de qué síntomas era necesario recurrir al médico? Aunque no existía un 
límite absoluto que definiera esta necesidad, los médicos reforzaban la idea de que la bibliografía dirigida a los especialistas no podía ser utilizada por personas que desconocían los términos técnicos. Reconocían que las madres que se guiaban por estos manuales para curar a sus hijos caían en profundos errores, estos libros "no sirven para el público, que no teniendo estudios especiales de medicina no puede poseer el indispensable criterio clínico, que solo lo dan la ciencia y la experiencia, de tal modo que se equivocan los síntomas y se confunden los cuadros sintomáticos, que pueden ser parecidos"(42). En consecuencia, por la voluntad de querer curar al niño, los padres podían caer en presunciones y no consultaban a los facultativos.

Los conocimientos médico científicos indicaban un proceso de formalización, aunque todavía poseían una baja complejidad. La falta de difusión de la pediatría convencional en la sociedad influyó en que cualquier persona podría asegurar el ejercicio de prácticas de curación y su eficacia resultaba a partir de tratamientos legitimados por la tradición. Los curanderos, sin instrucción alguna, se presentaban a la población como los únicos capaces de recetar medicamentos, apelando a nociones empiristas para simular las habilidades de los profesionales. Las aptitudes de los charlatanes, "no son ni siquiera discutidas por las inocentes víctimas. Se les considera dotados de una alta sabiduría, capaz de afrontar con éxito los más desesperados ca$\operatorname{sos}^{\prime \prime(43)}$. El sistema que validó la medicina infantil, y que se fundaba en derechos sobre el ejercicio de la disciplina, solo era permitido a quienes exhibían un título profesional que los capacitaba. Para el doctor Luis Morquio, el profesional "debe seguir estudiando y perfeccionándose, para satisfacer las dificultades que ofrece la asistencia en esta edad, se comprende cuan distante se estará de eso cuando intervienen personas incultas, analfabetas, que con sus procedimientos resultan más peligrosas que si el niño enfermo estuviera abandonado a su propia suerte" ${ }^{\prime(44)}$. El grado de experticia de los especialistas, que se adquiría en la práctica médica, iba ligado a las diferencias que configuraba parte de este campo: el curandero era declarado como alguien ignorante de las ciencias.

El emplazamiento de hospitales infantiles ocasionaría la transformación de los hábitos sobre la enfermedad, los especialistas señalaban que la única forma de combatir la mortalidad de los párvulos era la fundación de ese recinto. En estos lugares sería posible determinar el impacto que tuvieron las enfermedades durante el primer año de vida sabiendo "de una manera positiva dónde está el peligro que es preciso combatir"(45), para así conocer la distribución de cada una de ellas, medir el grado de letalidad y profundizar en los conocimientos sobre las patologías de los lactantes. Para el doctor Ricardo Dávila Boza, el problema era consecuencia de la ignorancia del pueblo en materia de enfermedades y la ausencia de elementos básicos, necesarios para la atención y la utilización en los enfermos. En efecto, "no hai por el momento manera de hacer que los niños sean bien atendidos en sus casas durante sus enfermedades, i no queda otro recurso que suplir esa deficiencia por la asistencia hospitalaria"(46).

¿Qué problemas hospitalarios debían resolver para hacer descender la mortalidad? A principios del siglo $\mathrm{XX}$, la medicina infantil se caracterizaba por "la superficialidad de los conocimientos respecto a la clínica pediátrica y en puericultura"(47). Se trataba de un ámbito en que la cantidad de especialistas no satisfacía la demanda. En 1894, la discusión parlamentaria abordó este tema y se centró en el hospital para niños de Valparaíso, destinado a menores, cuyo rango etario se hallaba entre los cuatro y siete años de edad, a pesar de que en esta edad los párvulos tenían mayor capacidad de resistencia y los más expuestos eran los niños desde su nacimiento hasta los cuatro años. Por esta razón, la Junta de Beneficencia del puerto solicitó recursos económicos para ampliar el establecimiento. En respuesta, el diputado Eduardo Edwards señaló que los terribles estragos de la mortalidad infantil se explicaban por la falta de un recinto en la capital destinado a velar por la salud de los párvulos, por consiguiente habría que dedicar "esos cien mil pesos, que 
piensan invertir en Valparaíso en un local para establecer un hospital para niños en Santiago, que vendría a llenar las más premiosas necesidades"(48).

Hasta entonces, la planificación del futuro recinto se sustentaba en argumentos políticos y técnicos. El diputado del Partido Radical, Carlos Robinet señalaba que el ingeniero Carlos Barroilhet tomaba "por modelo el hospital de Berlín, que reúne las condiciones más modernas sobre arquitectura hospitalaria" ${ }^{\prime(49)}$. En este período se pensaba que, naturalmente, era necesario un ingeniero para dirigir los trabajos de construcción, pero que en este proceso se debían considerar los puntos de vista de los integrantes de la Junta de Beneficencia.

Para cumplir con el anhelo de contar con un hospital infantil en Santiago, se recalcaban los efectos positivos que tendría un establecimiento de este tipo en los niños abandonados de la Sociedad Protectora de la Infancia. En "el año 1899, la mortalidad de párvulos en todo el año disminuyó en 300 defunciones. Esta cifra tan exacta, es un poderoso argumento a favor del hospital de niños"(50).

Si bien la propia comunidad médica, consciente de los brotes infecciosos que año a año provocaban gran cantidad de fallecimientos de niños, manifestaba la falta de un establecimiento destinado al tratamiento de estas enfermedades, un grave brote de sarampión en Santiago durante 1900 llevó a que el presidente de la Junta de Beneficencia y administrador del Cementerio General, Manuel Arriarán, solicitara al Ministerio del Interior la fundación de un hospital para niños. Para ello, convocó a facultativos para ejercer el cuidado de los infantes en una propiedad de la Congregación de las Hijas de San José Protector de la Infancia, localizada en la caIle Moneda con Almirante Barroso, que funcionaba como casa de ejercicios y donde la Junta de Beneficencia improvisó un hospital para niños.

Era un edificio descrito como amplio, higiénico, con extensos patios, comedores espaciosos, aposentos aseados, numerosos corredores, murallas sólidas y limpias. La propiedad tenía dos pisos: en el segundo habitaban las monjas que dirigían la casa y el primero se proyectó el hospital. Con una capacidad para 80 camas, el establecimiento disponía de una sala de aislamiento "destinada especialmente a las enfermedades de fiebre, a las derivaciones de la alfombrilla, a afecciones diversas"(51). También contempló una farmacia, en la que una religiosa preparaba las medicinas que se entregaban a los pacientes; una cocina donde se preparaban las dietas y comidas para los niños: "diariamente se consumen 12 decalitros de leche; unas cuantas arrobas de carne; una cantidad enorme de pan"(51). Dos veces por semana recibían la visita del doctor Roberto del Río y, diariamente, concurría Manuel Arriarán, cuya figura era comparada por las religiosas con la de Pedro Montt, durante la fundación de la Casa de Orates.

\section{EL HOSPITAL DE NIÑOS ROBERTO DEL RÍO}

Sin duda, la coyuntura de brotes infecciosos puso de manifiesto la necesidad de implementar un hospital para la atención infantil. El sitio elegido correspondía a un edificio localizado en la calle Matucana; el primer administrador fue Manuel Arriarán y su funcionamiento estaría a cargo de la Congregación de las Hijas de San José Protector de la Infancia. El hospital se construyó con la finalidad de organizar, de manera estable, la higiene en la infancia y su asistencia médica. La puesta en marcha de las atenciones requería ciertos ajustes y resolver problemas de carácter práctico. En una sesión del Consejo Superior de Higiene, el doctor Eugenio Cienfuegos señalaba que en el hospital solo se recibían niños afectados por enfermedades benignas, lo que incidió en las escasas cifras de mortalidad que registraba el recinto, "por no existir todavía el número suficiente de salas de aislamiento, no se admiten niños con difteria, los cuales se mandan, cuando su estado lo permite, previa inyección de serum, a otros hospitales, donde existen salas especiales para esta clase de enfermos"(52). En los primeros años de rodaje, los niños con afecciones contagiosas como la fiebre tifoidea, el 
coqueluche, la neumonía, la bronconeumonía o las meningitis, fueron derivados a los hospitales de adultos o eran rechazados.

Si se cruza el número de infantes fallecidos (Tabla 1) con los muertos en el hospital (Tabla 2), su comparación entrega porcentajes de atención en torno al $5 \%$ del total. Por ejemplo, en 1909, murieron 307 enfermos en el hospital, que equivalen al $3,69 \%$ de los 8.316 decesos en la provincia de Santiago durante ese año, lo que muestra que la difusión y la instauración del espacio todavía no cubría amplios sectores de la población.

En esa etapa, la articulación del trabajo hospitalario resultaba compleja porque no se disponía de los instrumentos para practicar cirugías; por ejemplo, las afecciones que requerían procedimientos quirúrgicos como las hernias, apendicitis, cálculos vesicales, quemaduras extensas o contusiones graves, no podían ser tratadas en los niños. Recién a fines de 1903 se realizaron las primeras intervenciones, con el apoyo de materiales entregados por la sección de cirugía del Hospital de San Vicente de Paul. La ausencia de normas de higiene en los pabellones y la posibilidad de adquirir infecciones durante el período posoperatorio hicieron que las cirugías fueran procedimientos riesgosos en las décadas de funcionamiento del recinto. En 1910, la Junta de Beneficencia, por indicación del médico Roberto del Río, aprobó $\$ 3.000$ pesos chilenos destinados a la instalación del servicio de autopsias, en una pieza al lado del actual depósito de cadáveres, con los útiles necesarios. Estos avances en los instrumentales contribuirían a la especialización de las funciones ejecutadas en el establecimiento y las mejoras en las observaciones aumentarían el grado de certeza en los diagnósticos de las enfermedades infantiles.

En esos años, el hospital de niños ocupaba un extenso terreno, disponía de patios y salas con buena ventilación. Entre las

Tabla 2. Movimientos del Hospital de Niños Roberto del Río, Santiago, 1908-1930.

\begin{tabular}{|c|c|c|c|c|c|}
\hline Año & $\begin{array}{c}\text { Existencia del año } \\
\text { anterior }\end{array}$ & Entradas & Salidas & Muertes & $\begin{array}{l}\text { Existencia para el } \\
\text { año siguiente }\end{array}$ \\
\hline 1908 & 279 & 2.670 & 2.350 & 307 & 292 \\
\hline 1909 & 292 & 2.240 & 1.952 & 307 & 273 \\
\hline 1910 & 273 & 2.253 & 1.972 & 276 & 278 \\
\hline 1911 & 278 & 2.433 & 1.898 & 190 & 345 \\
\hline 1912 & 345 & 2.932 & 2.561 & 334 & 382 \\
\hline 1913 & 312 & 3.295 & 2.933 & 324 & 350 \\
\hline 1914 & 350 & 3.230 & 2.992 & 282 & 306 \\
\hline 1915 & 306 & 3.441 & 3.177 & 274 & 296 \\
\hline 1916 & 293 & 3.419 & 3.182 & 3.452 & 260 \\
\hline 1917 & 260 & 3.694 & 3.199 & 3.666 & 288 \\
\hline 1919 & 187 & 4.976 & 4.097 & 691 & 375 \\
\hline 1920 & 375 & 3.593 & 3.237 & 503 & 228 \\
\hline 1921 & 228 & 4.203 & 9.930 & 981 & 285 \\
\hline 1922 & 361 & 3.497 & 3.237 & 355 & 266 \\
\hline 1923 & 266 & 3.310 & 2.863 & 432 & 281 \\
\hline 1924 & 281 & 3.309 & 2.965 & 425 & 290 \\
\hline 1925 & 290 & 3.120 & 2.756 & 354 & 300 \\
\hline 1926 & 300 & 2.842 & 2.515 & 315 & 312 \\
\hline 1927 & 312 & 3.194 & 2.808 & 398 & 300 \\
\hline 1928 & 300 & 2.469 & 2.208 & 277 & 284 \\
\hline 1929 & 286 & 2.489 & 2.484 & 363 & 291 \\
\hline 1930 & 291 & 2.719 & 2.275 & 420 & 315 \\
\hline
\end{tabular}

Fuente: Elaboración propia con base en los datos del Anuario Estadístico de la República de Chile, 1908-1930. 
principales innovaciones, contaba con una maquinaria para la descremación y esterilización de la leche. Las publicaciones destacaban la inclusión de una sala Ilamada "Cunas de Belén" o de las "mamitas", a ese lugar ingresaban los párvulos menores de dos años y eran atendidos por sus propias madres ( $\mathrm{Fi}$ gura 1), a quienes se les proporcionaba comida y alojamiento, frente a las respectivas cunas de los infantes. El recinto contaba con la "Sala de San José" destinada a la atención durante el período de convalecencia de la cirugía, en el que recibían medicinas. Anexo al edificio, estaba el dispensario para niños enfermos de poca gravedad, que podían curarse en sus casas. Esta sección era atendida por los doctores Luis Calvo Mackenna, Clemente Torretti y Francisco Jaramillo(53).

Sin embargo, los comienzos del hospital infantil generaron controversias entre los médicos, acerca de cuáles eran las condiciones que debía tener el establecimiento para el desarrollo correcto de sus actividades. Lo primero que llamaba la atención era que el destino original para el cual había sido construido el edificio era la Escuela Normal de Preceptores, es decir, una finalidad diferente al uso que se le estaba dando, por eso era calificado por la prensa como "...estrecho. En las salas hay un excesivo número de enfermos.
La lavandería está mal instalada"(54). En gran medida, se describía como un lugar de alta densidad, porque los niños hospitalizados que requerían lactancia estaban con sus madres, lo que obligaba a distribuir el espacio entre los pacientes y ellas. Desde el punto de vista de la higiene pública y para evitar contagios, una de las soluciones fue la edificación de salas de espera para clasificar a los enfermos, antes de enviarlos a salas específicas, según el tipo de patología. Paralelamente, se construyó "bajo la dirección de un especialista, una sala para enfermedades de ojos, nariz, oídos y garganta"(54).

En la práctica, estas transformaciones en la arquitectura hospitalaria enfrentaban varias dificultades. En primer lugar, el aumento constante de la población de Santiago originó más demanda y colapsos en los servicios asistenciales, sumado a que "las provincias cercanas al enterarse que la capital tiene un hospital de niños, las madres de por allá, sin entrar a averiguar lo que prescribe la higiene respecto al cubaje en estos recintos de niños, se dejan venir a buscar asilo"(54). Además, algunos niños eran trasladados porque requerían un tratamiento o para la ejecución de una intervención quirúrgica urgente. En segundo lugar, por las circunstancias indicadas, no era posible cerrar el local para ampliar sus

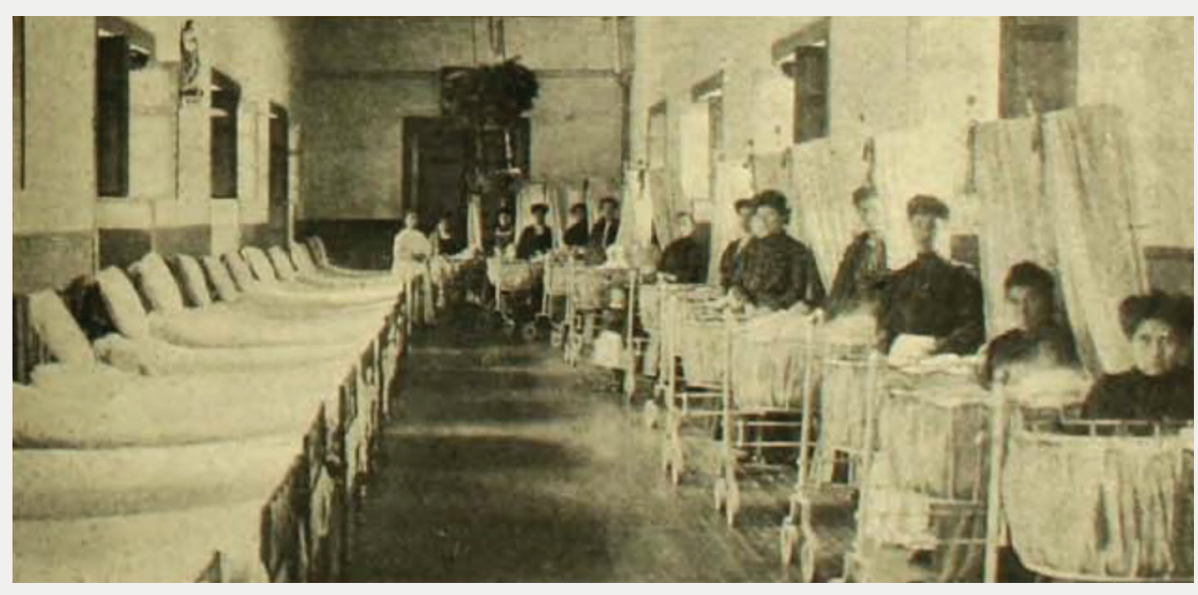

Figura 1. Sala "Cunas de Belén" o de las "Mamitas" en el Hospital de Niños, Santiago de Chile, 1909. Fuente: Revista Zig-Zag ${ }^{(53)}$. 
instalaciones y se disponía de los espacios de manera improvisada, con la disyuntiva entre el ideal científico -que ordenaba dar asilo a un número determinado de enfermos- y las exigencias de la caridad -que imponían no rechazar a los enfermos graves, sin otorgarles auxilio-, por lo tanto, desde el principio fue una necesidad construir otro hospital de niños en Santiago.

En cuanto a su funcionamiento, el cuidado de los infantes estuvo a cargo de las religiosas. A pesar de que los médicos elaboraban los diagnósticos y realizaban evaluaciones diarias a los enfermos, ellas se relacionaban cotidianamente con los infantes internados. En ese régimen, durante las visitas de los padres, según la religiosa Esperanza González, tenían la costumbre de "Ilevarles a sus niños toda clase de golosinas, algunas de lo más indigestas; pero es un hábito que no les es posible impedir, pues bastaría para que esta gente, de tan escasos conocimientos, viese en esto un peligro para sus hijos"(55). El conocimiento del contenido de las dietas muestra que se entregaba cualquier tipo de alimentación a los enfermos, sin considerar las afecciones, lo que podría provocar el recrudecimiento de alguna dolencia digestiva.

El Anuario Estadístico fija, desde 1911, las características del personal dedicado al funcionamiento del recinto. Dada la reiteración de los datos, la muestra considera tres años precisos. Según la Tabla 3, disminuyó el número de médicos internos, pero aumentó el de los especialistas contratados externamente. A inicios de la década de 1920, se requirió un profesional que estuviera a cargo de la farmacia y que efectuara la preparación de los medicamentos; durante ese período, la cantidad de religiosas se mantuvo estable.
Por último, la planta de trabajadores estaba compuesta por un grupo heterogéneo de personas, algunos consignados bajo la categoría de practicantes y otros como empleados, que aumentaron durante la década de 1910 hasta llegar al máximo a inicios de la década siguiente y desde ahí comienzan a disminuir de modo progresivo.

A partir de la Tabla 3 es posible extraer dos elementos de análisis. En primer lugar, el número total de trabajadores que accedía a la educación formal, en 1911, era de 26 individuos, frente a los 88 que provenían de espacios legos o considerados practicantes de alguna disciplina científica. En 1929, los médicos y farmacéuticos sumaban 35, en cambio, las monjas y otros empleados eran 60. Si bien en ambos puntos de la muestra se advierte que los funcionarios de apoyo eran más que los especialistas, las proporciones experimentan cambios. Los médicos en 1911, 1921 y 1929 equivalían al 22,8\%, $10,1 \%$ y $36,8 \%$ respecto de la planta de empleados. En segundo lugar, la cantidad total de personal en esos años estuvo en 114, 128 y 95 individuos, o sea, se mantuvo en torno a los 100. Al relacionar estas cifras con el número de niños ingresados al recinto (Tabla 2), se aprecia que, a medida que aumentó la demanda durante la década de 1910, también crecieron las contrataciones de practicantes y otros empleados. Por otro lado, entre los años 1921 y 1929 ambas variables decrecen, lo que puede haber ocurrido porque, en 1919, el Hospital de Niños Manuel Arriarán inicia la atención al público.

En esta época, quedan en evidencia las tensiones entre los legos y los médicos en la administración de los recintos hospitalarios. Hasta ahí, la Junta de Beneficencia era la

Tabla 3. Distribución de personal del Hospital de Niños Roberto del Río, Santiago de Chile, 1911-1929.

$\begin{array}{cccccc}\text { Año } & \text { Médicos internos } & \text { Médicos externos } & \text { Farmacéuticos } & \text { Monjas } & \begin{array}{c}\text { Practicantes y otros } \\ \text { empleados }\end{array} \\ 1911 & 5 & 21 & - & 14 & 74 \\ 1921 & 2 & 10 & 1 & 15 & 100 \\ 1929 & 2 & 32 & 1 & 15 & 45\end{array}$

Fuente: Elaboración propia a partir de datos del Anuario Estadístico de la República de Chile, 1911-1929. 
encargada de la organización, atribuciones y competencias en los hospitales. Los vecinos de la ciudad eran los encargados de la dirección de hospitales, cementerios y manicomios, situación defendida por la experiencia de sus componentes, ya que "no es preciso ser facultativo como sostienen algunos; basta ser persona de buen criterio, de posición independiente y de conciencia honorable ${ }^{\prime \prime(56)}$. Esta estructura orgánica fue cuestionada, a medida que fueron especializándose y progresando los conocimientos científicos, se requerían médicos para la articulación de sus funciones. De manera progresiva, los hospitales fueron diferenciando los médicos, los legos y las religiosas. El hospital se transformaba en un espacio de trabajo, donde se consolida la autoridad médica, convirtiéndose en un lugar con propósitos terapéuticos para la población. En consecuencia, se impone, paulatinamente, la reorganización de los espacios a cargo de la gestión de los médicos, lo que redunda en una mayor recepción de los avances científicos de un campo del conocimiento en formación ${ }^{(57)}$. En esa línea, en 1917, la Facultad de Medicina y Farmacia envió una carta al Ministerio del Interior, proponiendo mejoras en el ámbito hospitalario y cambios en el reglamento orgánico vigente desde 1886, para lo cual "uno de los dos administradores del establecimiento debe ser médico. Los múltiples servicios necesitan ser organizados y vigilados por una persona que tenga la suficiente preparación técnica"(58). En la comunidad científica se estaba modificando la finalidad de los hospitales: no solo debían asilar, sino que también debían medicar para curar y devolver al sujeto sano a la sociedad.

La creación de estos recintos no significaba una modificación automática de las representaciones de la población sobre la enfermedad, la sola instalación no promovía que la gente acudiera con sus niños a estos lugares. Por eso, la Junta de Beneficencia acordó "hacer propaganda por todos los medios posibles a fin de convencer que la gente debe ir en busca de los hospitales i de las dispensarías para mejorar, i que deben observar estrictamente las prescripciones del médico"(46). Solicitarían a la prensa, los sacerdotes y la policía la difusión de esta información e instalar en la sociedad la necesidad de trasladar a los niños enfermos durante las primeras fases de las afecciones. La recomendación principal consistía en llevar a los niños al hospital, en cuanto fueran observados los síntomas de un malestar, para averiguar a cuál corresponde, sin esperar nuevas etapas de la enfermedad. En sentido contrario, las monjas del hospital de niños de la Casa de San José, señalaban que "únicamente morían los enfermitos que llegaban en el último período de la enfermedad. No había caso de algún niño llevado con oportunidad que hubiese fallecido"(46), la prontitud en presentarse ante el médico debía ser el primer remedio que las madres suministraban a sus chicos.

\section{CONCLUSIONES}

Ingresar en la densidad cultural de las patologías infantiles permite apreciar varios escenarios en el proceso de validación de una materia científica. Un porcentaje de la población relacionaba la mortalidad con la religión, de ahí surge la importancia que adquiría el ritual del angelito, la ilegitimidad o el abandono de párvulos en las iglesias. Más allá de este sustrato, la relación de la higiene, como factor que disminuiría la probabilidad de contraer una enfermedad y el contagio por vía de los gérmenes, no estaba del todo desarrollada, los médicos calificaban a la población, una y otra vez, como ignorante en estas prácticas. En cuanto a la prevención de las afecciones infantiles, tardaría varias décadas en legitimar un conjunto científico de saberes sobre las enfermedades que afectan a las personas en esta etapa de la vida, lo que no corresponde a una dinámica en línea recta o sencilla, más bien, es una transformación compleja que involucra el desarrollo de una disciplina científica profesional y su encuentro con elementos sociales y culturales.

En ese contexto, el itinerario terapéutico que tenía como punto inicial los cuidados en el hogar, continuaba con las observaciones del curandero y, luego se concurría a los 
dispensarios. En esa época, los consejos de las personas cercanas, validados por la experiencia y la cantidad de medicamentos suministrados a los infantes, constituían, probablemente, un paliativo para los malestares, pero en ningún caso eran la cura definitiva, incluso, podían llegar a resultar contraproducentes para la salud. La población requería tratamientos que habían demostrado curar una enfermedad $y$, en este momento, los curanderos tenían una amplia red de contactos en la comunidad, las opiniones de las personas recomendaban y aseguraban la eficacia de sus métodos. En parte, esto explica la agresiva campaña que impulsan los médicos, junto con las autoridades y la justicia, contra lo que denominaban seres despiadados, que cometían el delito de ejercer ilegalmente la profesión. Por esta razón, fue necesario llevar a cabo una serie de mecanismos para normar y limitar el grado de especialización de un área de la medicina.

La inserción de la cátedra de Medicina Infantil se enmarca en la profundización de las disciplinas y la legitimación de saberes que transformaron los programas de formación académica. Como toda dinámica que involucra el progreso de los conocimientos científico-médicos, en sus inicios, la cátedra de enfermedades de la infancia mantuvo una discusión inmersa en el espacio académico, influenciada y sustentada por los debates que se estaban produciendo en Europa. Sin embargo, a fines del siglo XIX, esta situación experimentó cambios a partir de múltiples factores: las mejoras de las estadísticas demográficas permitieron visualizar la mortalidad infantil como un problema social, los brotes anuales de infecciones se cobraron miles de vidas, el aumento de población deterioraba los servicios entregados por los dispensarios, la salubridad de la capital era paupérrima y los conventillos solo se multiplicaban. En esas circunstancias, la construcción de un hospital dedicado a tratar las enfermedades de los niños tenía carácter de urgencia.

La instalación de un hospital de niños marca un hito, que trae consigo los albores de la medicina infantil al país, y que se condice con los primeros indicios de la difusión de los conocimientos científicos sobre la salud y la enfermedad en los párvulos. A su vez, la Escuela de Medicina se alza como una instancia de discusión académica, acerca de las causales que explican las muertes en el período de la niñez. Establecer la relación de los médicos con las madres se convirtió en un proceso complejo, que consistía en instalar la idea de que, ante los síntomas de una enfermedad, era necesario trasladar al párvulo al recinto. Los hospitales marcaron una nueva conducta, desde el tratamiento de las afecciones en el hogar, hacia estas instituciones y constituyen los inicios de espacios destinados a enfrentar las elevadísimas tasas de mortalidad a través del desarrollo de la pediatría. En ese contexto, cuando se produjo la medicalización de la infancia, estas instituciones y los médicos consiguieron ampliar su autoridad científica y legitimar su área de trabajo entre la población.

\section{AGRADECIMIENTOS}

Agradecemos el financiamiento otorgado por la Comisión Nacional de Investigación Científica y Tecnológica (CONICYT), a través de su programa formación de capital humano avanzado: Becas de Doctorado Nacional otorgadas en 2010 y 2015. Agradecemos los Comentarios de los evaluadores de este artículo y el trabajo editorial de la revista Salud Colectiva.

\section{REFERENCIAS BIBLIOGRÁFICAS}

1. Instituto Nacional de Estadística. Estadísticas Vitales: Anuario 2014 [Internet]. 2014 [citado 5 may 2020]. Disponible en: https://tinyurl.com/y9w7kc4c.
2. Naciones Unidas. Anuario Estadístico de América Latina y el Caribe [Internet]. 2014 [citado 14 feb 2020]. Disponible en: https://tinyurl.com/yad23z5a.

3. Vargas N. Historia de la pediatría chilena: Crónica de una alegría. Santiago: Editorial Universitaria; 2002.

4. Orrego Luco A. Recuerdos de la Escuela. Buenos Aires: Editorial Francisco de Aguirre; 1976.

5. Cruz Coke R. Historia de la Medicina Chilena. Santiago: Editorial Andrés Bello; 1995.

6. Laval E. La epidemia de sarampión de 1899-1900 en Chile y la creación del primer hospital de niños de Santiago. Revista Chilena de Infectología. 2002;19(2):121123. doi: 10.4067/S0716-10182002000200012. 
7. Ávila Cisneros I, Padrón Puyou F, Frenk S, Rodríguez Pinto M, (coords.). Historia de la pediatría en México. Ciudad de México: Fondo de Cultura Económica; 1997.

8. Baeza Bacab MA. Orígenes de la pediatría institucional: el Hospital de Maternidad e Infancia de la Ciudad de México en el siglo XIX. Boletín Médico del Hospital Infantil de México. 2017;74(1):70-78. doi: 10.1016/j. bmhimx.2016.10.010.

9. Cavieres E. Ser infante en el pasado: Triunfo de la vida o persistencia de estructuras sociales, la mortalidad infantil en Valparaíso, 1880-1950. Revista de Historia Social y de las Mentalidades. 2001;5(1):31-58

10. Llanos Reyes C, Lanfranco MF. La discusión política sobre la mortalidad infantil en Chile durante la década de 1930: Elementos para una aproximación histórico política. Anuario de Estudios Americanos. 2017;74(2):675703. doi: 10.3989/aeamer.2017.2.10.

11. Chávez Zúñiga $P$, Soto Lara JJ. Historia de la mortalidad infantil en la Casa de Huérfanos de Santiago (1898-1923). Anuario de Estudios Americanos. 2019;76(2):645-677. doi: 10.3989/aeamer.2019.2.09.

12. Chávez Zúñiga $P$, Soto Lara JJ. La primera estadística sanitaria infantil de la provincia de Santiago de Chile (1860-1929). Dynamis. Historia de la Medicina. 2019;39(1):149-174.

13. Chávez Zúñiga P. Ilegitimidad, alcoholismo y tuberculosis: explicaciones médicas de la mortalidad infantil. Santiago de Chile (1870-1912). Nuevo Mundo Mundos Nuevos. 2019. doi: 10.4000/nuevomundo.76861.

14. Chávez Zúñiga $P$, Soto Lara JJ. Mortalidad infantil en Santiago: representaciones y discursos, Chile, 1860-1914. História, Ciências, Saúde-Manguinhos. 2018;25(4):12811300. doi: 10.1590/s0104-59702018000500023.

15. Chávez Zúñiga P. La mortalidad infantil en las viviendas: las consecuencias de la migración campociudad en Santiago (Chile, 1865-1930). Revista Temas Americanistas. 2018;40:265-286.

16. Zárate MS. Dar a luz en Chile, siglo XIX: De la "ciencia de la hembra" a la ciencia obstétrica. Santiago: Ediciones Universidad Alberto Hurtado; 2007.

17. Zárate MS. De partera a matrona: Hacia la asistencia profesional del parto en Chile en el siglo XIX. Calidad en la Educación. 2007;27:283-297.

18. Zárate MS. Proteger a las madres: origen de un debate público, 1870-1920. Revista Nomadías. 1999;1:163182.

19. Correa MJ. ¿Quiénes son los profesionales? Justicia, profesionalización y ejercicio médico en el Chile urbano de la segunda mitad del siglo XIX. Dynamis. 2017;37(2):273-293

20. Brieger G. Bodies and borders: a new cultural history of medicine. Perspectives in Biology and Medicine. 2004;47(3):402-421. doi: 10.1353/pbm.2004.0044.
21. Lucier $P$. The professional and the scientist in nineteenth-century America. ISIS. 2009;100(4):699-732. doi: 10.1086/652016.

22. Abbott A. The system of professions: an essay on the division of expert labor. Chicago: University of Chicago Press; 1988.

23. Torstendahl R, Burrage M. The formation of professions: knowledge, state and strategy. London: Sage; 1990.

24. Pickering A. Science as practice and culture. Chicago: University of Chicago Press; 1992.

25. Mohr J. Doctors and the law: Medical jurisprudence in nineteenth-century America. Baltimore: Johns Hopkins University; 1993.

26. Armus D. La enfermedad en la historiografía de América Latina moderna. Asclepio. 2002;54(2):41-60. doi: 10.3989/asclepio.2002.v54.i2.140.

27. Birn AE. Child health in Latin America: historiographic perspectives and challenges. História, Ciências, Saúde-Manguinhos. 2007;14(3):677-708. doi: 10.1590/ S0104-59702007000300002.

28. Mazzeo V. La mortalidad de la primera infancia en la ciudad de Buenos Aires en el período 1860-2002. Papeles de Población. 2007;13(53):241-272.

29. Calvo Mackenna L. Lo que deben saber las madres para criar bien a sus hijos : cartilla de Puericultura al alcance del pueblo. En: Primer Congreso Nacional de Protección á la Infancia: Trabajos y Actas. Santiago: Imprenta Barcelona; 1913.

30. Coni E. La mortalidad infantil en la ciudad de Buenos Aires: Estudio comparativo con la mortalidad infantil de Rio de Janeiro, Montevideo, Lima, Méjico, y otras ciudades americanas [Internet]. Buenos Aires: Imprenta de Pablo E. Coni, especial para obras; 1879 [citado 10 may 2019]. Disponible en: https://tinyurl.com/y8e3q5s9.

31. Genta Mesa G. Anotaciones para una historia de la pediatría y la puericultura. latreia. 2006;19(3):296-304.

32. Huard P, Laplane R. Histoire illustrée de la pédiatrie. París: Les Éditions Roger Dacosta; 1981.

33. Salas E. Historia de la medicina en Chile. Santiago: Imprenta Vicuña Mackenna; 1894.

34. Zorrilla P. Hijiene: Reseña sobre las causas de la mortalidad de los niños en Santiago, i medios de evitarlas - Comunicación de don Pablo Zorrilla a la Facultad de Medicina. Anales de la Universidad de Chile [Internet]. 1861;18(1) [citado 10 may 2019]. Disponible en: https://tinyurl.com/ydyk4on8.

35. Sociedad Médica. Reforma del plan de estudios médicos. Revista Médica. 1881;X(4):122-125.

36. Vargas C. Movimiento de la población en Chile. Mortalidad de párvulos. Santiago: Memoria de prueba para optar al grado de Licenciado en la Facultad de Medicina i Farmacia. Universidad de Chile; 1882. 
37. Mc Lean S. The modern hospital. Sección Revista Extranjera, traducción del doctor Alfredo Commentz. Revista de Beneficencia. 1918;X(5):58-64.

38. Consejo Superior de Protección a la Infancia. Memoria anual. Santiago: Imprenta Nacional; 1916.

39. Lisboa Huerta G. Mortalidad de párvulos en Chile: sus causas, modo de evitarlas. [Memoria de prueba para el examen de Licenciado en la Facultad de Medicina]. Santiago: Universidad de Chile; 1886.

40. Dávila Boza R. Mortalidad de los niños en Santiago sus causas i sus remedios: Informe presentado a la dirección del Instituto de Hijiene. Revista Chilena de Higiene. $1899 ; \mathrm{V}: 265-371$.

41. Hidalgo W. Medicina doméstica de la infancia o sea consejos a las madres sobre el modo de criar, cuidar i educar i curar a sus hijos por sí mismas. Santiago: Imprenta de la Librería del Mercurio; 1877.

42. Torres I. Cómo tener y criar hijos sanos y robustos. Santiago: Editorial Nascimiento; 1926.

43. Mortalidad infantil: Acción de los curanderos, la palabra de un médico. Las Últimas Noticias, 25 oct 1908. Localizado en: Sección periódicos, Salón Camilo Henríquez, Biblioteca Nacional, Santiago.

44. Morquio L. El problema de la mortalidad infantil. Montevideo: Consejo Nacional de Higiene, Oficina de Educación y Propaganda; 1931.

45. Melfi M. Mortalidad infantil y protección a la infancia. [Memoria de prueba para optar al grado de Licenciado en la Facultad de Medicina y Farmacia]. Santiago: Universidad de Chile; 1907.

46. Obra nacional: Hospitales para niños. El Chileno, 7 oct 1900. Localizado en: Sección periódicos, Salón Camilo Henríquez, Biblioteca Nacional, Santiago.

47. La mortalidad infantil: Medio interesante empleado en la lucha contra ella. El Mercurio, 3 ago 1906. Localizado en: Sección periódicos, Salón Camilo Henríquez, Biblioteca Nacional, Santiago.
48. Cámara de Diputados. Sesión $58 .^{\text {a }}$ ordinaria en 29 de agosto de 1894. Localizado en: Biblioteca del Congreso Nacional de Chile, Santiago.

49. Cámara de Diputados. Sesión $8 .^{a}$ estraordinaria en 10 de noviembre de 1900. Localizado en: Biblioteca del Congreso Nacional de Chile, Santiago.

50. Hospital de niños: Urgencia de establecerlo. El Mercurio, 13 sep 1900. Localizado en: Sección periódicos, Salón Camilo Henríquez, Biblioteca Nacional, Santiago.

51. Una visita al hospital de niños de Santiago. El Chileno, 27 oct 1900. Localizado en: Sección periódicos, Salón Camilo Henríquez, Biblioteca Nacional, Santiago.

52. El Hospital de Niños. El Mercurio, 10 sep 1903. Localizado en: Sección periódicos, Salón Camilo Henríquez, Biblioteca Nacional, Santiago.

53. Hospital de Niños [Internet] Revista Zig-Zag. 24 jul 1909;(231):49-50 [citado 5 may 2020]. Disponible en: https://tinyurl.com/y8xm5zfs.

54. El Hospital de Niños: Sus defectos, manera de corregirlos. El Chileno, 26 sep 1903. Localizado en: Sección periódicos, Salón Camilo Henríquez, Biblioteca Nacional, Santiago.

55. Nuestros establecimientos de beneficencia: El Hospital de Niños de la avenida Matucana. El Mercurio, 16 ago 1916. Localizado en: Sección periódicos, Salón Camilo Henríquez, Biblioteca Nacional, Santiago.

56. La Junta de Beneficencia. El Diario Popular, 13 jul 1903. Localizado en: Sección periódicos, Salón Camilo Henríquez, Biblioteca Nacional, Santiago.

57. La mortalidad en Santiago. El Mercurio, 6 ene 1908. Localizado en: Sección periódicos, Salón Camilo Henríquez, Biblioteca Nacional, Santiago.

58. Organización de la Beneficencia Pública. El Mercurio, 8 ago 1917. Localizado en: Sección periódicos, Salón Camilo Henríquez, Biblioteca Nacional, Santiago.

\section{FORMA DE CITAR}

Chávez Zúñiga P, Brangier Peñailillo V. Esa diaria gotera de la muerte: Mortalidad infantil y los albores de la medicina pediátrica en la provincia de Santiago, Chile, 1870-1913. Salud Colectiva. 2020;16:e2727. doi: 10.18294/ sc. 2020.2727 .

Recibido: 16 feb 2020 | Versión final: 4 abr 2020 | Aprobado: 8 abr 2020 | Publicado en línea: 16 may 2020

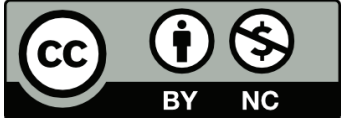

Esta obra está bajo una licencia de Creative Commons Reconocimiento-NoComercial 4.0 Internacional. Reconocimiento - Permite copiar, distribuir y comunicar públicamente la obra. A cambio, se debe reconocer y citar al autor original. No Comercial - Esta obra no puede ser utilizada con finalidades comerciales, a menos que se obtenga el permiso. 